\title{
Implementation of Computerized Physician Order Entry in Primary Care: A Scoping Review
}

\author{
Inge Dhamanti iD ${ }^{\text {I-3 }}$ \\ Eva Kurniawati (iD ${ }^{4}$ \\ Elida Zairina ${ }^{5}$ \\ Ida Nurhaida iD ${ }^{6}$ \\ Salsabila Salsabila ${ }^{1,2}$ \\ 'Faculty of Public Health Universitas \\ Airlangga, Surabaya, East Java, Indonesia; \\ ${ }^{2}$ Center for Patient Safety Research, \\ Universitas Airlangga, Surabaya, \\ Indonesia; ${ }^{3}$ School of Psychology and \\ Public Health, La Trobe University, \\ Melbourne, VIC, Australia; ${ }^{4}$ Department \\ Cardiothoracic Surgery, Maastricht \\ University Medical Center, Maastricht, \\ The Netherlands; ${ }^{5}$ Faculty of Pharmacy, \\ Universitas Airlangga, Surabaya, \\ Indonesia; ${ }^{6}$ Faculty of Computer Science, \\ Mercu Buana University, Jakarta, \\ Indonesia
}

\begin{abstract}
Purpose: This scoping review aimed to assess the implementation and outcomes of computerized physician order entry (CPOE) in primary care.

Methods: A scoping review was carried out in accordance with the Joanna Briggs Institute's guidelines (JBI). The databases PubMed, CINAHL, Science Direct, and Google Scholar were all searched. The full text of each article was reviewed for eligibility after the title and abstract were evaluated. JBI data extraction were used to extract data. Donabedian's framework served as the foundation for the data discussion.

Results: Based on the inclusion criteria, seven studies were included. The studies' main goal in common was to analyze the outcome or impact of implementing CPOE systems in ambulatory or primary care settings. Several studies described the framework, current state of implementation, and evaluation or recommendation following CPOE system implementation. Many positive effects were felt by physicians or prescribers, pharmacists, patients, and primary care providers, with patient safety being the primary goal.

Conclusion: Although this study discovered some issues and factors associated with CPOE implementation and adoption, such as infrastructure, workflow, level of engagement, and safety culture, CPOE has many positive outcomes for patients, physicians, and primary care. To improve CPOE adoption in healthcare, particularly primary care, more research into the structure, framework, and components of CPOE deployment is required.
\end{abstract}

Keywords: e-prescription, computerized physician order entry, patient safety, problem, adoption

\section{Introduction}

Patient safety is the essential goal of all healthcare organizational levels. Patient safety in primary care includes the prevention of errors, adverse outcomes, and harm to patients related to primary care healthcare. ${ }^{1}$ Patient safety also refers to how well patients are safeguarded from avoidable harms. In both developing and developed countries, up to $25 \%$ of the general population experiences harm while receiving care in primary care settings. ${ }^{2-4}$

The most common types of incidents in primary care were associated with medication and diagnostic errors. ${ }^{5}$ Severity level of the incidents in primary care can be classified into no harm, mild harm, moderate harm, severe harm, and death. ${ }^{6}$ Issues contributing to compromised patient safety in primary care settings include errors in diagnosis, prescription, communication breakdown, unsafe medication practices, fragmentation of care and error in clinical decision making. ${ }^{5,7}$ A large study that analysis the National Reporting and Learning System from primary care identified medication-related incidents caused less harms compared to incident
Correspondence: Inge Dhamanti Email inge-d@fkm.unair.ac.id 
related to clinical decision-making incidents, that caused the most serious patient harm outcomes. ${ }^{7}$ Medicationrelated errors is the most common type of incident in primary care, which are preventable or avoidable. ${ }^{8}$ Burden of ADEs in primary care included admissions to hospital, length of hospital stay and deaths related to ADEs occurring in primary care. ${ }^{9}$

The utilization of health information systems, such as a computerized physician order entry (CPOE) could reduce patient safety incidents related to medications at the primary care level. CPOE is the process of electronically entering medication orders or other physician instructions ${ }^{10}$ however some CPOE systems only allow physicians and nurse practitioners to prescribe medications and send the prescriptions to the pharmacy electronically. ${ }^{11}$ CPOE automated the ordering process, resulting in orders that are more readable, complete, and standardized, including prescriptions. ${ }^{8}$ Results can be reviewed and all orders, including admission orders, prescriptions, investigations, and care needs, can be entered using CPOE systems. ${ }^{12}$ Prescribing medication using CPOE systems can eliminate many medicationrelated safety incidents in primary care. The use of CPOE systems in conjunction with clinical decision support also provides dose recommendations, reduces illegible orders, aids with calculations, and screens for allergies and medication interactions. Primary care providers save time handwriting prescriptions and reduce the mental workload by utilizing CPOE systems. $^{11}$

Patient safety in primary care remains a neglected issue that has received less attention than it has in hospital settings. $^{13}$ The national and international patient safety agenda is still primarily focused on hospitals. ${ }^{2}$ Furthermore, because CPOE systems are typically implemented in hospital settings and are still uncommon in primary care, studies focusing on primary care are required. We conducted a scoping review in this study to assess the implementation and outcomes of CPOE in primary care settings.

\section{Materials and Methods}

This study was conducted using a scoping review as outlined by the Joanna Briggs Institute (JBI) for conducting a scoping review. ${ }^{14}$ Scoping review methodologies were used to determine the implementation of CPOE and the outcomes of CPOE implementation in primary care. The protocol for this scoping review was developed based on the PRISMA-P guidelines. ${ }^{15}$

\section{Search Strategy}

The following electronic databases were searched on August 2021: PubMed, CINAHL, Science Direct, and Google Scholar. Keywords and Boolean operators ("OR" and "AND") related to the implementation of CPOE systems in primary care were used in the search (see Table 1). The inclusion criteria were original articles written in English, articles published from 2016 to 2021, studies conducted in the primary care setting, and studies with any outcomes related to the implementation of CPOE will be considered.

\section{Eligibility Criteria and Data Selection}

Eligibility was defined as studies that clearly described the implementation of CPOE in primary care or ambulatory care. Furthermore, studies were included if the outcome after the implementation of CPOE was objectively defined. A total of 1469 articles were retrieved and 25 duplications were automatically removed using Mendeley

Table I Literature Search Strategy

\begin{tabular}{|l|l|l|l|}
\hline \multirow{2}{*}{ Search } & \multicolumn{3}{|c|}{ Field } \\
\cline { 2 - 4 } & \multicolumn{1}{|c|}{ Population } & \multicolumn{1}{|c|}{ Concept } & \multicolumn{2}{|c|}{ Context } \\
\hline \#I AND \#2 AND \#3 & Primary care & $\begin{array}{l}\text { Computerized physician } \\
\text { order entry }\end{array}$ & Implementation \\
\cline { 2 - 4 } & Public health center & $\begin{array}{l}\text { Computerized provider } \\
\text { order entry }\end{array}$ & \\
\cline { 2 - 4 } & Ambulatory care & $\begin{array}{l}\text { Care provider order } \\
\text { entry }\end{array}$ & \\
\cline { 2 - 4 } & & e-prescribing & \\
\hline
\end{tabular}




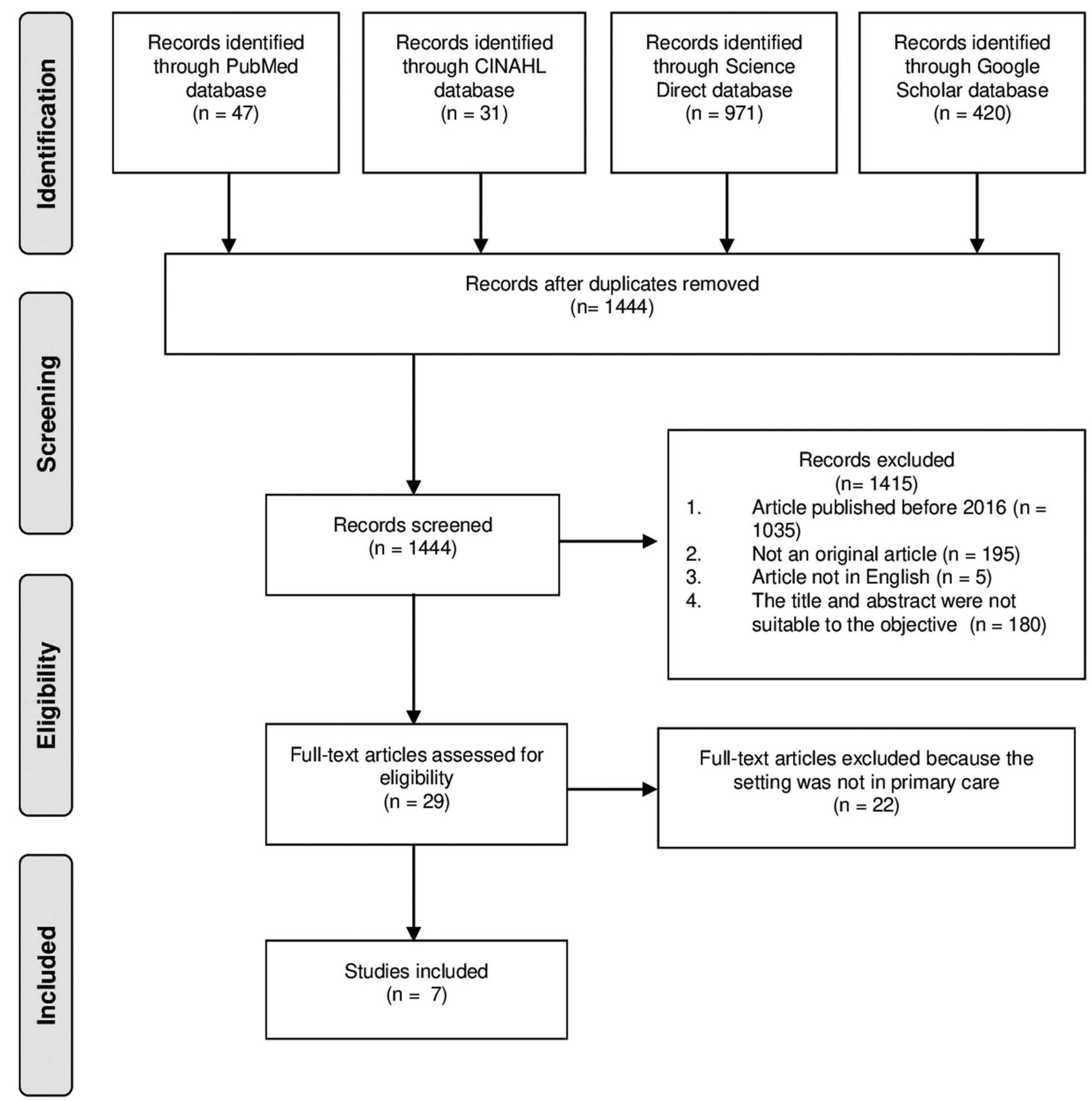

Figure I Prisma flow diagram.

Reference Manager. After duplicates were removed, there were 1444 records left. The eligibility of 29 full-text articles was determined. Seven publications passed the eligibility process. Figure 1 shows a full illustration of the study flow.

\section{Data Extraction and Synthesis}

Data extraction was carried out in accordance with the methodology provided by the $\mathrm{JBI},{ }^{14}$ and this study was used the broad population, concept and context (PCC) framework recommended by the JBI for Scoping Reviews to determine the search strategy (could be seen in Table 1). ${ }^{16,17}$ Information including the authors, publication year, country of origin of the study, and study characteristics (setting, study design, aim of the study, significant findings, and outcomes) were retrieved. We analysed the key findings of the studies using Donabedian's framework. 


\section{Results}

The final review was comprised of seven papers (Figure 1). Most of the studies were conducted in North America. Five studies were conducted in the United States, ${ }^{8,11,18-20}$ one study was conducted in Canada, ${ }^{21}$ and one study was conducted in Turkey. ${ }^{22}$ The CPOE studies were extracted using Donabedian's settings (structure), processes, and outcomes. ${ }^{23}$ Structures were defined as the equipment, resources, or framework aspect of the primary care setting while implementing CPOE. Processes were defined as mechanisms or performers within the implementation of CPOE in primary care. The outcome or state after implementing the CPOE in primary care was defined as the implementation's impact or consequences.

\section{Study Characteristics}

Most of the studies were cross-sectional ${ }^{11,18,19,21,22}$ and only two of them categorised as quasi-experimental. ${ }^{8,20}$ The main context of the studies was similar, but the objectives varied. Two studies extended the scope of the CPOE beyond medication prescription, ${ }^{8,18}$ while the rest focused on electronic prescribing ${ }^{1,19-22}$ The main common objective within the studies was to analyze the outcome or impact after implementing CPOE systems in the ambulatory or primary care settings. ${ }^{8,11,20,22}$ Furthermore, several studies described the framework, current state of implementation, and evaluation or recommendation after implementing the CPOE system. ${ }^{18,19,21}$ The studies' outcomes were as follows: measured potential and preventable ADEs, the cause of preventable ADEs, prescription rates, CPOE use and the percent of CPOE use, positive effects of e-prescriptions, the problems involved in e-prescription writing, the level of satisfaction, adoption of e-prescribing, problems on the prescribers' and receivers' sides, identification of inappropriate and appropriate prescription content, and compliance rate. A detailed extraction of the studies is provided in Table 2 .

\section{CPOE Implementation in Primary Care}

The included studies used a wide variety of terms to describe CPOE, including "computerized prescribing," "computerized prescriber order entry," "e-prescribing," "e-prescription," and "electronic prescription." Of the seven studies, only one provided clear definitions of the framework or component to analyse the implementation of CPOE in primary care. Using Bell's framework, the study compared the computerized prescription system functions available at the time of the study in two study sites. ${ }^{8}$ The computerized prescription step using Bell's framework consisted of five steps: prescribe, transmit, dispense, administer, and monitor. Each prescription step had some functional capability that was applied while implementing the CPOE system in primary care. The functional capabilities at the prescribe step include patient section or identification, diagnosis selection and diagnosis-based reminders, medication selection menus, and safety alerts based on drug-choice errors due to allergies, formulary adherence, and dosage calculation. The second step was transmitting, which is the process of data transmission to the inpatient, retail, and pharmacy. The dispense step occurs when the physician dispenses the drugs, and drugchoice errors by physicians often occur at this step. The administer step has some functional capabilities, including patient education, medication administration aids, refill and renewal reminders, and drug stock reminders. The final step is to monitor step, which consists of administering automated patient questionnaires to detect adverse effects, follow-up contact, corollary prescriptions, and alerts for patient failure to refill.

The implementation of CPOE systems in primary care in the United States has increased, although the adoption rate remains low. ${ }^{18}$ Once CPOE systems are adopted in primary care, they become the dominant form of ordering and are used for more than $94 \%$ of orders. One included study showed that the adoption of the e-prescribing feature lagged both on the prescriber and pharmacy side. ${ }^{21}$ The low adoption rate was due to the system's poor quality from the user's perspective. Various issues can hinder the introduction of CPOE in primary care. In one study, the problem experienced by family physicians while using e-prescription was related to failure to maintain the infrastructure. The most common issues involved internet connectivity, computer (hardware) failure, program malfunction, and a lack of information on the user's part. As a result, physician training and infrastructure maintenance are critical in preventing errors in e-prescription writing and speeding up the process.

\section{Outcomes of CPOE Use in Primary Care}

The use of CPOE results in increased detection of potential and preventable adverse drug events (ADEs) caused by medication errors. ${ }^{8}$ Furthermore, the use of CPOE in conjunction with basic decision support and patient education has been linked to a reduction in the number of potentially avoidable adverse events (ADEs). The most common 


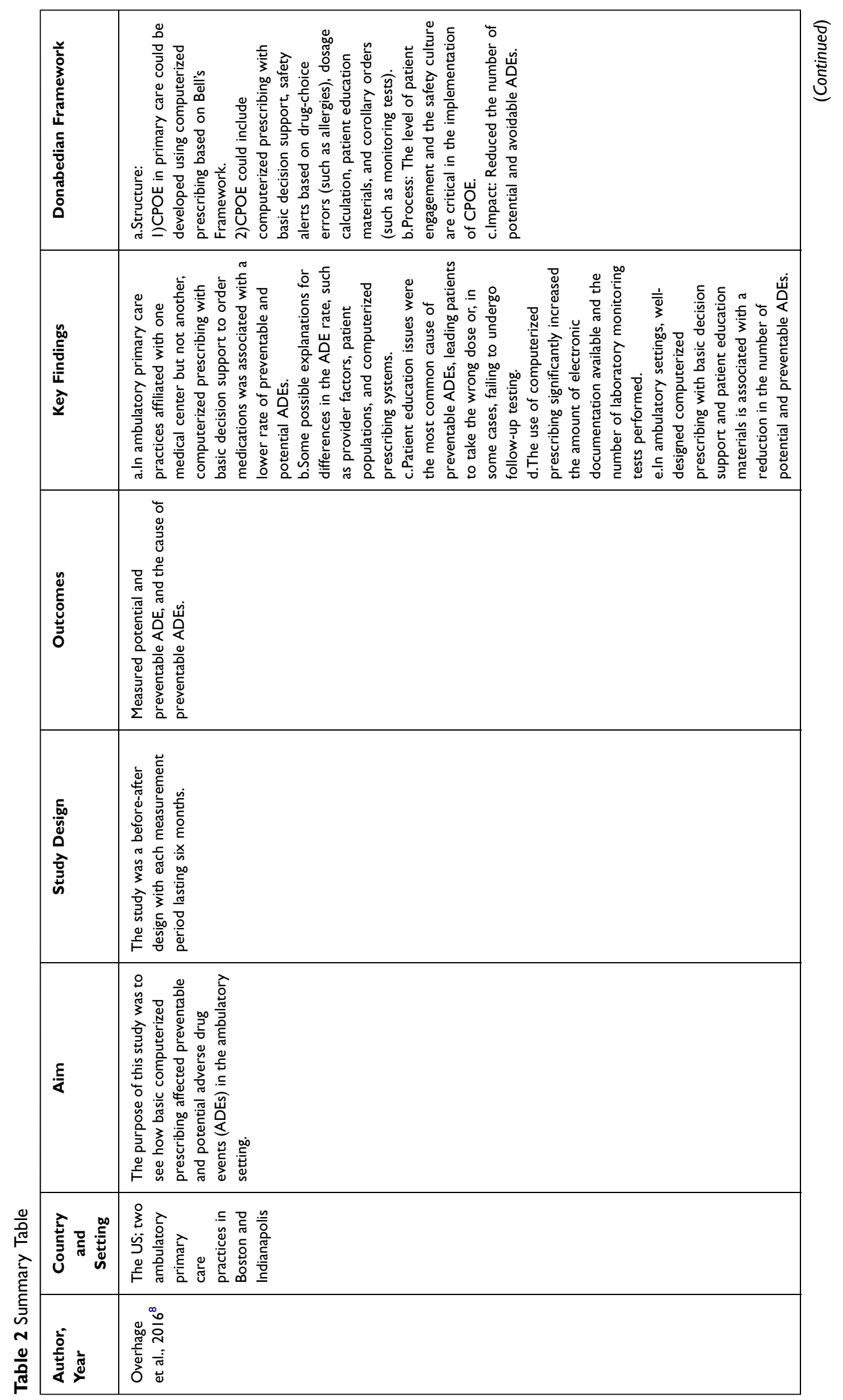




\begin{tabular}{|c|c|c|c|}
\hline 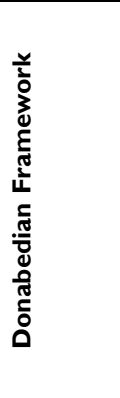 & 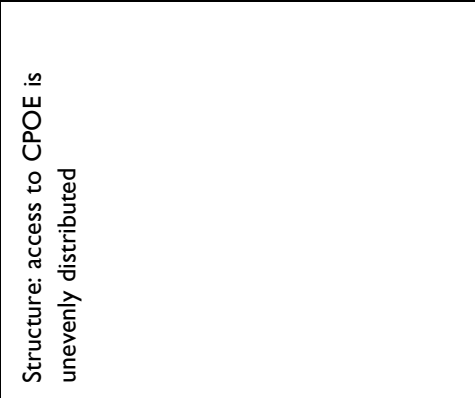 & 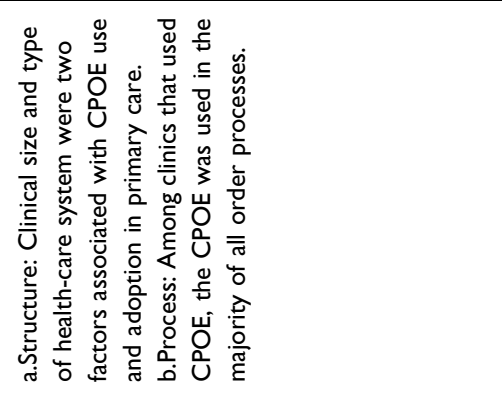 & 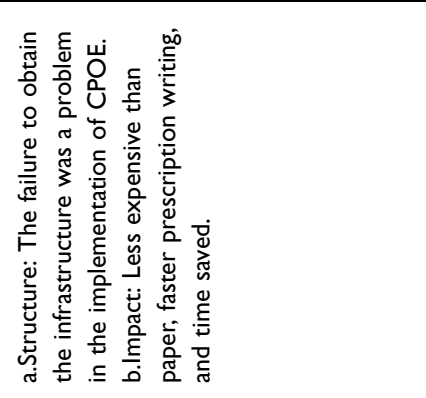 \\
\hline 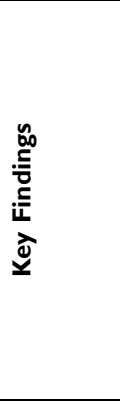 & 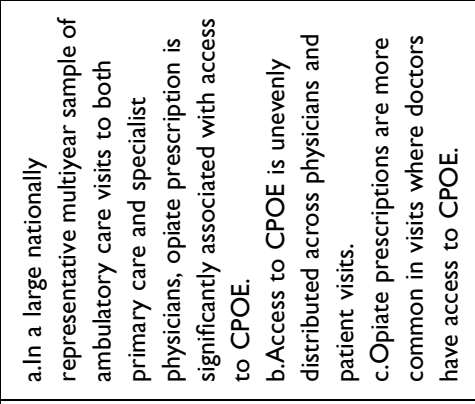 & 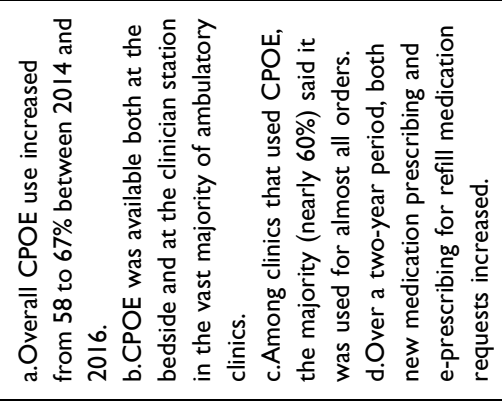 & 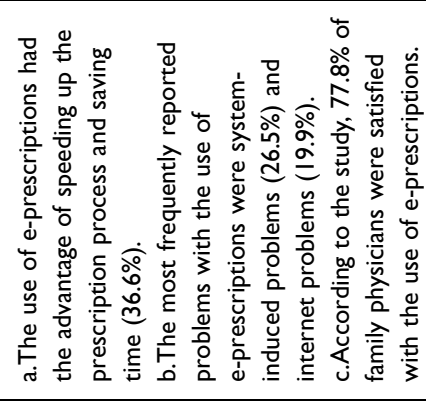 \\
\hline & 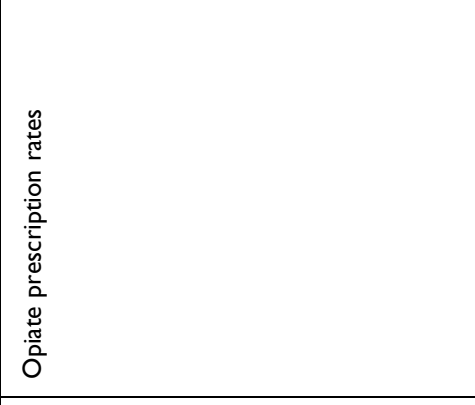 & 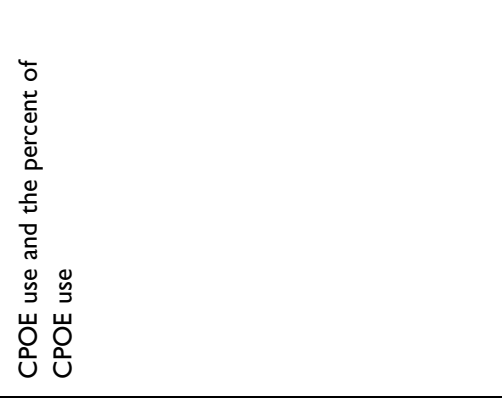 & 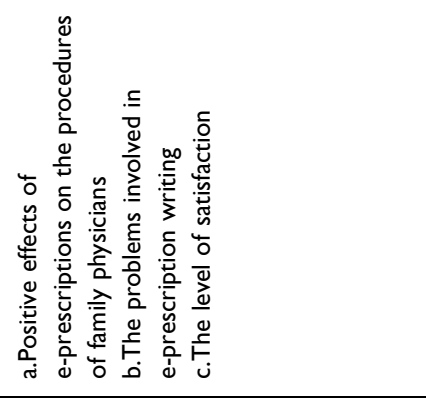 \\
\hline 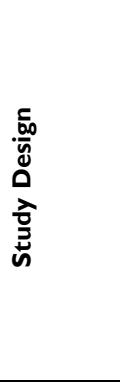 & 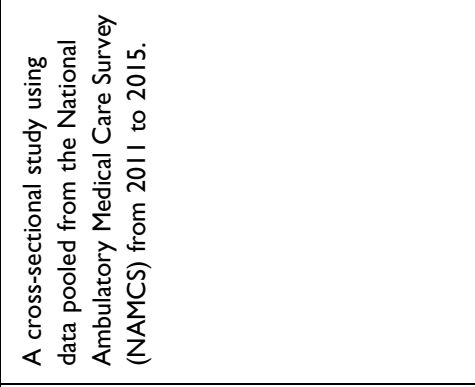 & 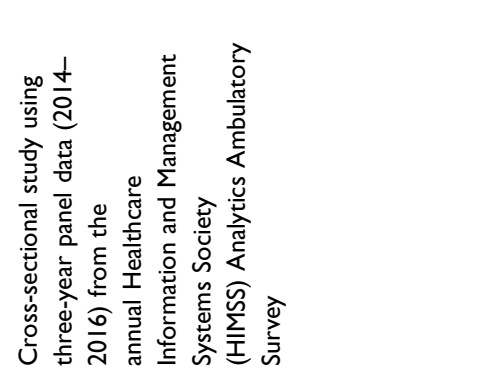 & 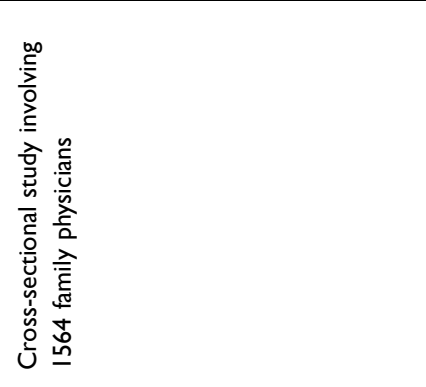 \\
\hline$\frac{\xi}{<}$ & 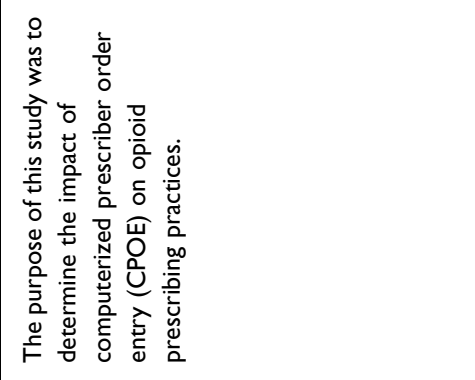 & 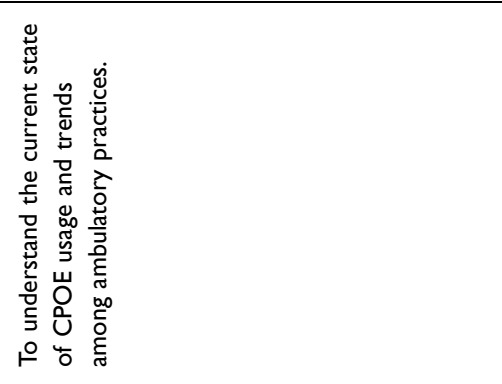 & 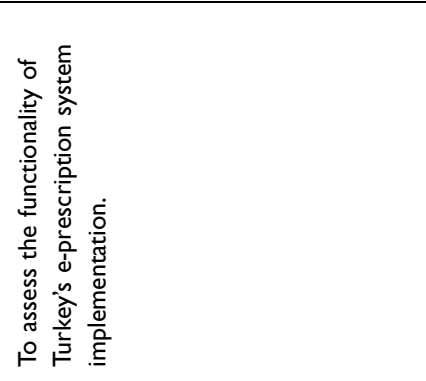 \\
\hline 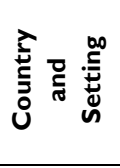 & 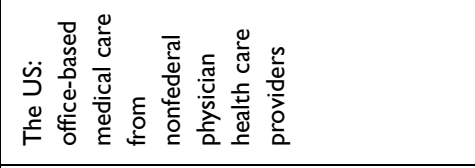 & 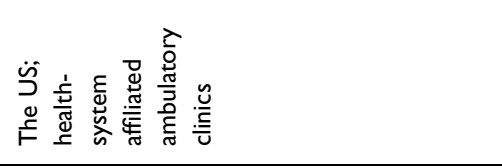 & 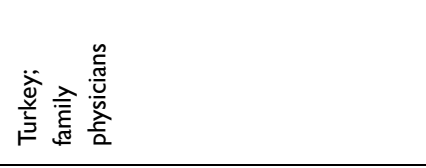 \\
\hline 豙 & 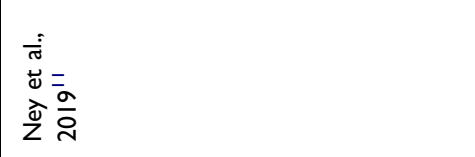 & 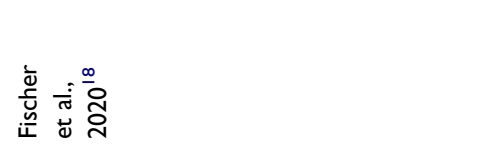 & 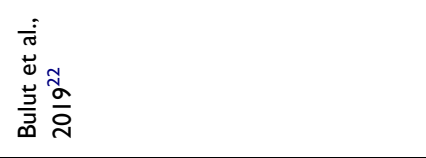 \\
\hline
\end{tabular}




\begin{tabular}{|c|c|}
\hline 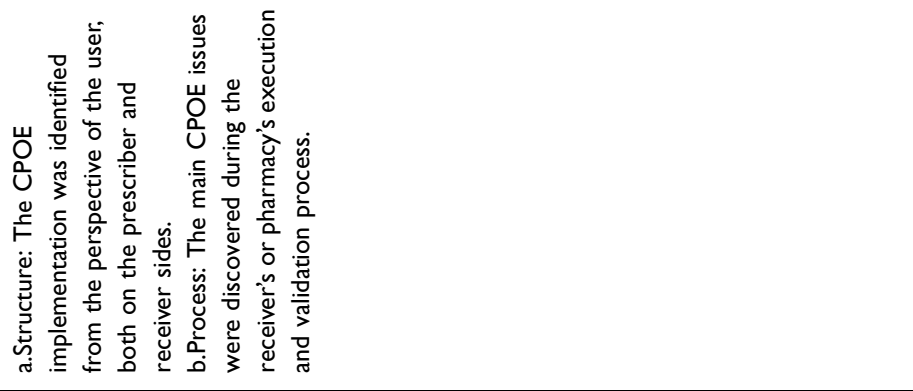 & 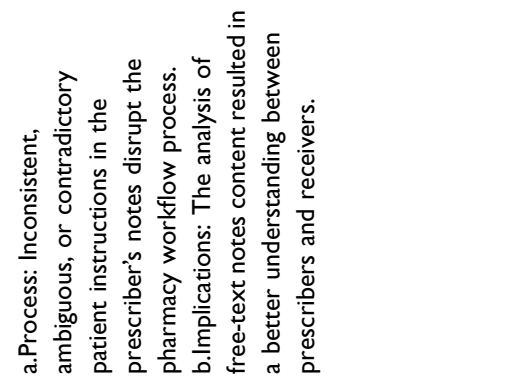 \\
\hline 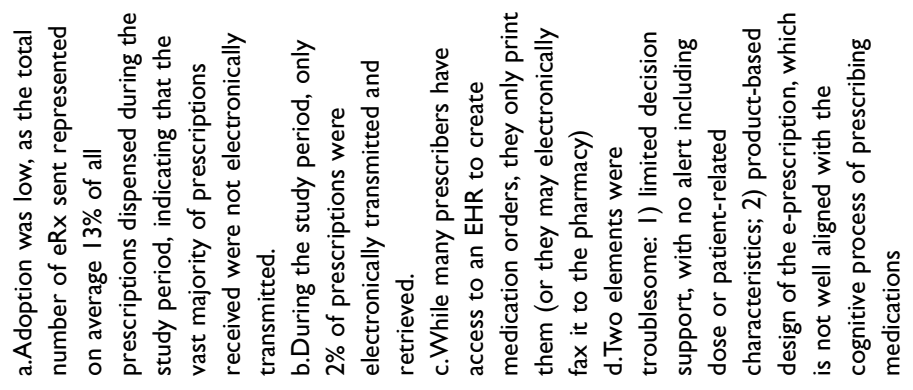 & 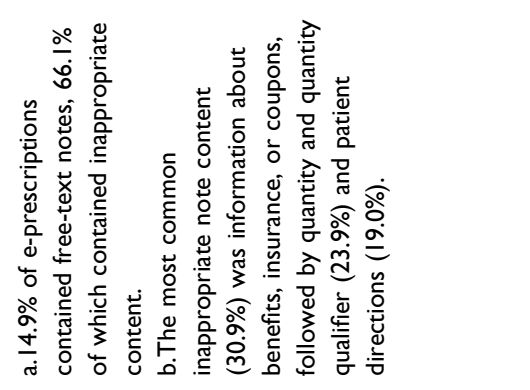 \\
\hline 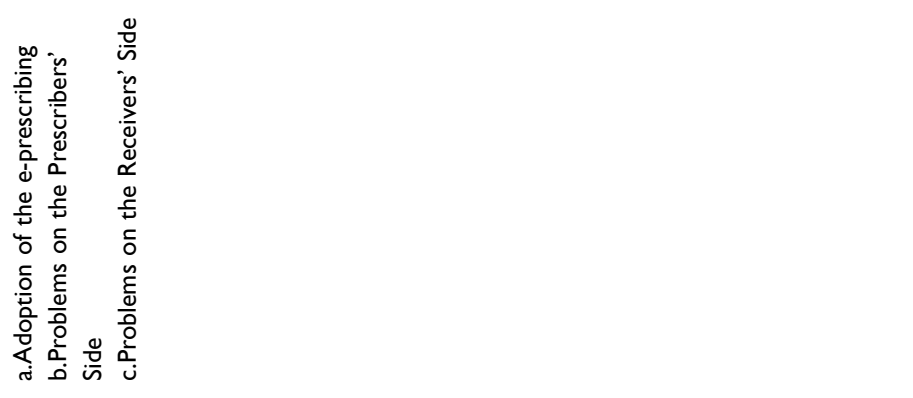 & 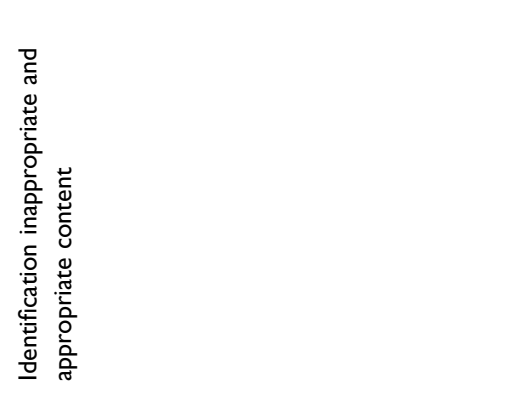 \\
\hline 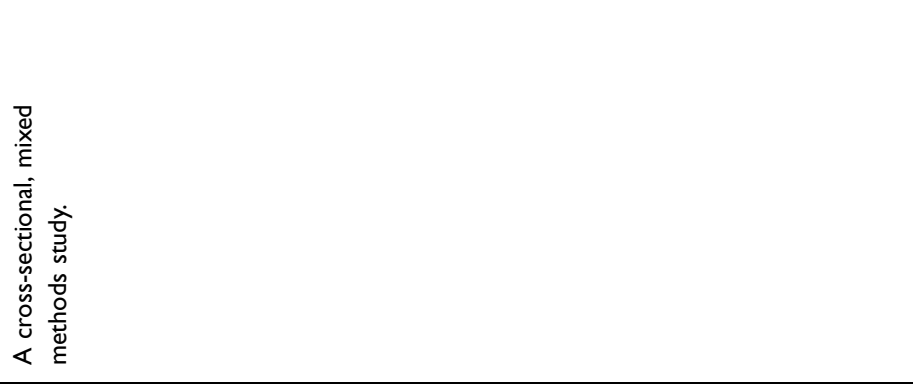 & 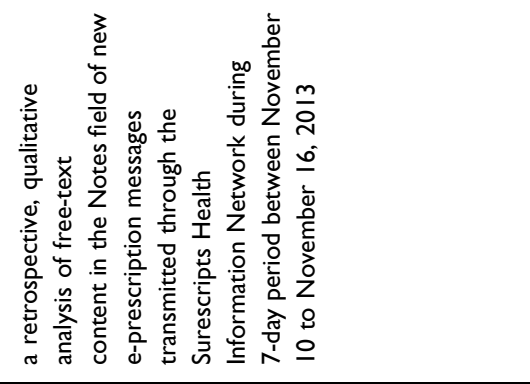 \\
\hline 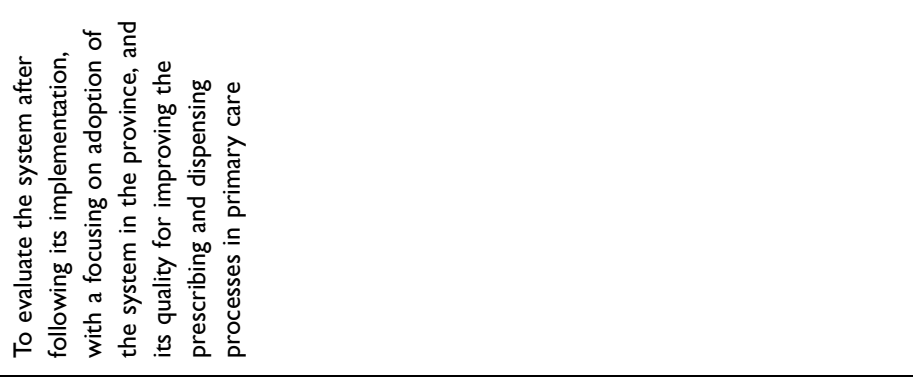 & 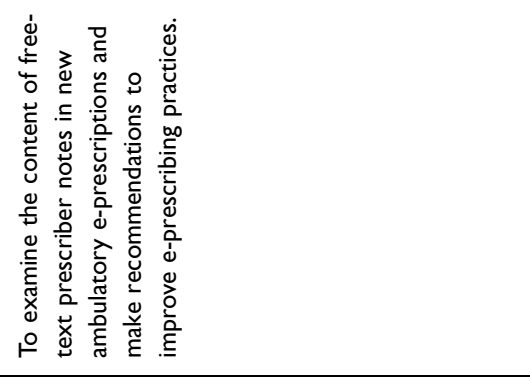 \\
\hline 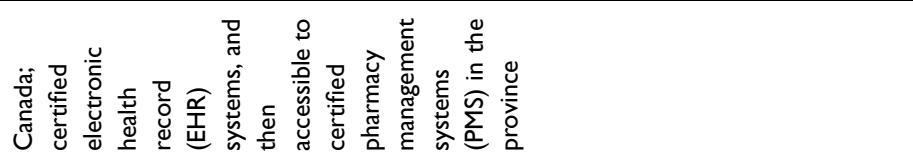 & 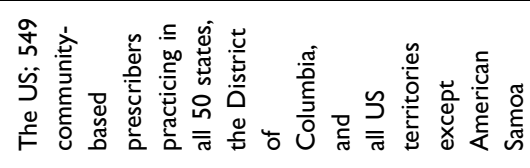 \\
\hline 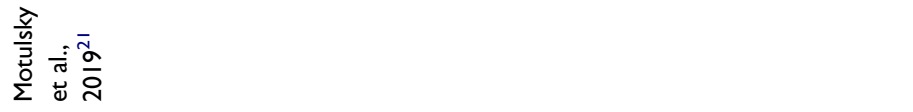 & 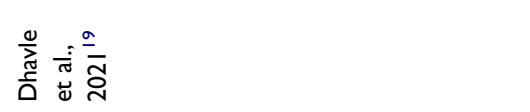 \\
\hline
\end{tabular}




\begin{tabular}{|c|c|}
\hline 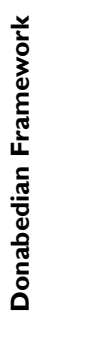 & 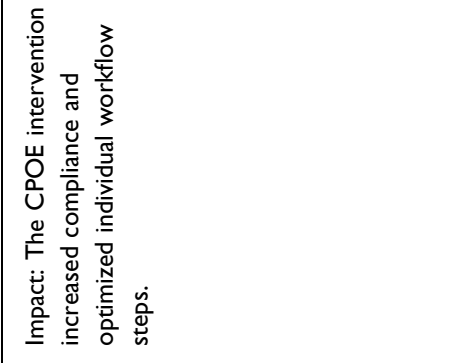 \\
\hline 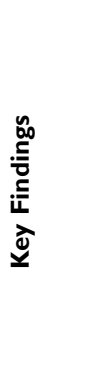 & 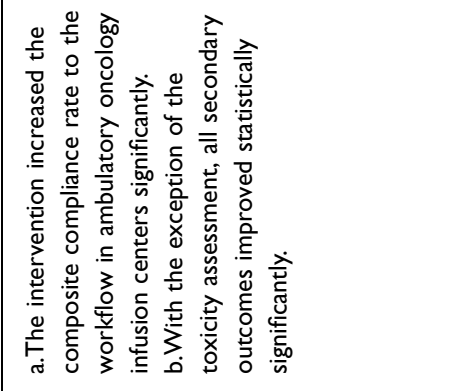 \\
\hline 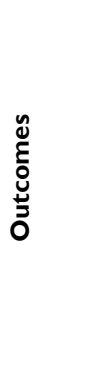 & 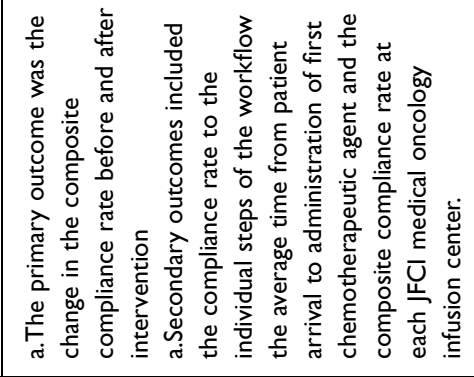 \\
\hline 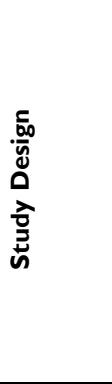 & 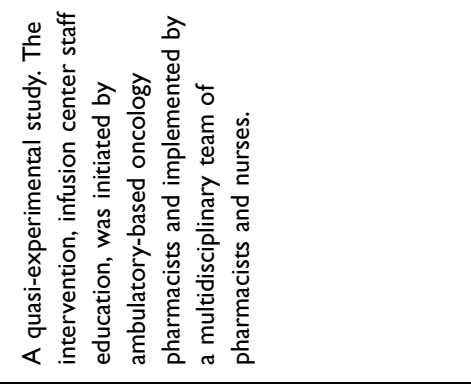 \\
\hline$\frac{\varepsilon}{<}$ & 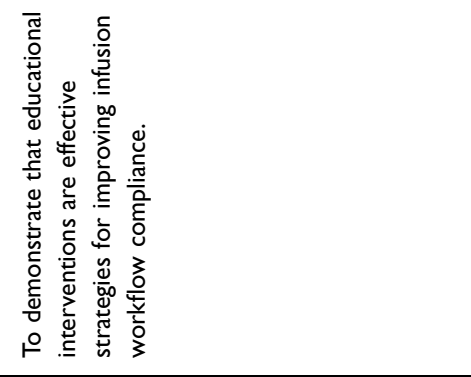 \\
\hline 害焉 & 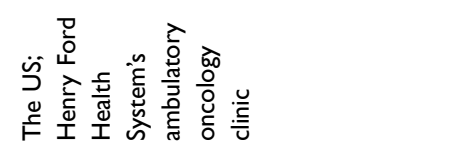 \\
\hline 竎㐫 & 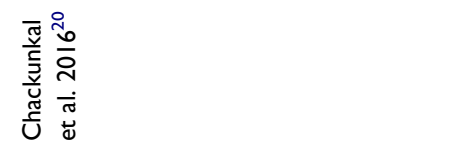 \\
\hline
\end{tabular}

cause of preventable ADE was a lack of patient education, which led to patients taking the wrong dose or, in some cases, failing to undergo follow-up testing.

Electronic documentation provided by CPOE systems assists providers in identifying errors in the prescribing process. CPOE or e-prescriptions could be generated faster than manual prescriptions, freeing up time for other tasks and processes, simplifying the addition of explanations, and speeding up decision-making. The use of e-prescriptions also reduced prescription writing errors and made e-prescriptions legible, exact, and comprehensive, according to the family physicians. ${ }^{22}$ Thus, e-prescription systems provided convenience for pharmacists to read prescriptions and reduced incorrect medicine or dosage errors. Despite these positive effects, other factors were associated with CPOE adoption. According to the included study, the size and type of primary care health-system were two related factors of CPOE adoption. ${ }^{18}$

The individual workflow step compliance rate, the average time from patient arrival to administration, and the composite compliance rate all improved after CPOE implementation. ${ }^{20}$ Another finding from the studies was concerning the issues of CPOE use. The main problems with CPOE can be identified from the perspectives of both the prescriber and the receiver. The prescribers' side was related to the medication order design and the lack of clinical decision support, whereas the receivers' side was related to the use of paper copy prescriptions. ${ }^{21}$ Another study emphasizes the significance of the appropriate content free-text notes field in e-prescriptions in order to avoid misunderstandings between prescribers and recipients. Inappropriate content can cause ambiguity or conflict between prescribers and recipients, causing the pharmacy workflow process to be disrupted. ${ }^{19}$

\section{Discussion}

This study focused on the implementation of CPOE systems in primary care. We discussed the study's findings using Donabedian's framework, consisting of structure, process, and outcome. Furthermore, we examined the infrastructure required to implement $\mathrm{CPOE}$ within the organization; analysis of the process highlighted problems or strategies within the implementation. Finally, we studied the outcome, impact, or consequences of CPOE adoption for patients, health professionals, and other stakeholders. Some lessons learned from the implementation of CPOE in primary care were discussed. E-prescription systems increase patient safety; the 
limitations and problems are related to its cost-related infrastructure and adoption by health facilities and clinicians.

Analysis of the results using Donabedian's approach revealed several key issues for primary care providers who plan to utilize CPOE systems. In terms of settings (structure), primary care providers must determine the framework and infrastructure needed before implementing the CPOE. Furthermore in terms of input factors clinic size and health-system need to be considered in the CPOE adoption, and also prescriber's and receiver's sides need to be considered in dealing with CPOE issues. ${ }^{18,21}$ The CPOE framework consists of steps and functional capabilities of each step, which serve as a guideline to assist the physician in effective implementation. The e-prescription system improves prescribing efficiency by implementing functional features to improve drug management. ${ }^{24}$ Aside from that the integrated functional CPOE system with basic decision support, safety alerts, and corollary orders increases as the functional capabilities increase. ${ }^{8}$ Thus, the CPOE framework is critical and must be fully developed and understood before implementing CPOE in primary care.

The infrastructure must be developed prior to deploying the CPOE system. According to the results of this study, the reliance on computers during the implementation of CPOE is one of the drawbacks of e-prescription systems. ${ }^{25}$ Computer-related problems included slowness, failure to get an internet connection, failure of computers (hardware) to work, and failure of the program (software) developed for e-prescribing to function. Both the computer hardware and software need to be maintained to avoid errors in the entire e-prescription process, because all data are connected.

This study revealed that CPOE was used in most of all orders process among clinics that were using it, but there were some problems found in the execution process of CPOE. The difficulties of implementing CPOE in primary care must be anticipated from a process standpoint. According to research, difficulties in CPOE implementation are most likely caused by physician latency, both on the prescriber and pharmacy side. ${ }^{21}$ The main issues on the prescriber's side were related to the design of the medication order, the absence of clinical decision assistance, the absence of an electronic prescription request feature, and the systematic printing of paper prescription copies. Meanwhile, the biggest issue at pharmacies was the challenge to adapt their workflow to an electronic prescription.
During the CPOE deployment phase, primary care providers must anticipate addressing those difficulties.

The level of engagement and safety culture among patients, prescribers, and receivers was critical in the implementation of the CPOE process. Throughout the implementation phase, physician training may help to reduce errors. Previous research suggests that physician training is required to improve knowledge of the e-prescribing system, so that the system does not appear as difficult. ${ }^{26}$ According to additional research, teaching new physicians how to use e-prescription systems reduces errors and speeds up system implementation. ${ }^{27}$ When using CPOE, training is required to improve physician comprehension, reduce errors, and speed up the prescription process. This corresponds to the findings that larger health-system sizes and health-system types that conduct patient education and staff training are associated with high CPOE adoption. ${ }^{18}$

A standardized procedure for CPOE implementation is required in all orders processed by clinics or primary care practices that use it. Most likely, the CPOE or e-prescription implementation process contains internally inconsistent, unclear, or incomplete information, preventing correct and efficient prescription processing and dispensing. ${ }^{28}$ As a result, a free-text note prescriber is required for the communication of appropriate e-prescription clinical notes with additional patient-specific information pertinent to the prescription and can be used by prescribers to make changes to or discontinue existing medications. Thus, the free-text note prescriber within the CPOE deployment is required for dealing with these issues because prescribers' inability to transmit information in a standardized manner can have serious consequences for patient safety. ${ }^{19,29}$

This study highlighted several positive outcomes or impacts of CPOE implementation in primary care, such as increased detection of potential and preventable ADEs, decreased patient safety incidents in primary care, improved compliance rate and prescription process, and optimization of individual workflow steps. ${ }^{8,11,20}$ Using CPOE improves physician satisfaction and reduces physician errors during the prescription process. ${ }^{22}$ Several other studies have shown that more than $80 \%$ of physicians are satisfied with e-prescription systems because they reduce paperwork throughout the prescription process and improve patient safety ${ }^{30}$ and improve prescription safety and accuracy. ${ }^{31}$ CPOE reduced the possibility of malpractice claims and pharmacy call-backs due to prescription errors caused by the doctor's handwriting. In terms of patient and pharmacist satisfaction, CPOE systems reduce 
patient wait time, eliminate the problem of ripping or losing paper prescriptions, reduce medication or dose errors, and simplify the medication acquisition process for patients. ${ }^{32}$ CPOE is more cost-effective than paper prescriptions because it drastically reduces the amount of paper and toner needed for prescriptions. ${ }^{33}$ Overall, e-prescribing systems improve healthcare service quality, increase the efficiency of prescribing and delivering pharmaceuticals, and reduce prescription errors and healthcare costs. $^{34}$

\section{Limitations}

There are several limitations to this study that must be acknowledged. Because only a few keywords were used in this study, the findings may have been limited. Because databases and papers published in English were used, potentially valuable studies on CPOE deployment in other languages may have been overlooked. Furthermore, there have been few recent original studies on the use of CPOE in primary care, resulting in a lack of data. However this study added updated information about CPOE implementation in primary care (within 5 years) and an analysis of the CPOE implementation using the Donabedian framework.

\section{Conclusion}

The implementation and outcomes of CPOE in primary care settings were assessed in this scoping review. This study discovered some issues and factors related to CPOE implementation and adoption, such as infrastructure, workflow, level of engagement, and safety culture. Despite implementation issues, CPOE has many positive outcomes such as reducing potential and preventable ADEs, increasing compliance rates, improving physician satisfaction, speeding up prescription writing, and saving time, paper, and costs. To improve CPOE adoption in healthcare, particularly primary care, more research into the structure, framework, and components of CPOE deployment is required.

\section{Acknowledgments}

We thank Universitas Airlangga for providing funding for this project.

\section{Disclosure}

The authors report no funding and no conflicts of interest for this work.

\section{References}

1. MH Al L, Dennis S, Short SD, Abdulhadi NN. Patient safety and safety culture in primary health care: a systematic review. BMC Fam Pract. 2018;19(1):1-12. doi:10.1186/s12875-018-0793-7

2. Michel P, Brami J, Chanelière $M$, et al. Patient safety incidents are common in primary care: a national prospective active incident reporting survey. PLoS One. 2017;12(2):1-14. doi:10.1371/journal.pone.0165455

3. Word Health Organization. Patient Safety. Available from: https:// www.who.int/news-room/fact-sheets/detail/patient-safety. Accessed July 8, 2021.

4. Auraaen A. The economics of patient safety in primary and ambulatory care. OECD Heal Work Pap. 2018;106:96.

5. Kuriakose R, Aggarwal A, Ramandeep K, Gambhir RS. Patient safety in primary and outpatient health care. J Fam Med Prim Care. 2020;9(1):548.

6. Cooper J, Williams H, Hibbert P, et al. Classification of patient-safety incidents in primary care. Bull World Health Organ. 2018;96(7):498505. doi:10.2471/BLT.17.199802

7. Cooper A, Edwards A, Williams H, et al. Sources of unsafe primary care for older adults: a mixed-methods analysis of patient safety incident reports. Age Ageing. 2017;46(5):833-839. doi:10.1093/ageing/afx044

8. Overhage JM, Gandhi TK, Hope C, et al. Ambulatory computerized prescribing and preventable adverse drug events. J Patient Saf. 2016;12(2):69-74. doi:10.1097/PTS.0000000000000194

9. Elliott RA, Camacho E, Jankovic D, Sculpher MJ, Faria R. Economic analysis of the prevalence and clinical and economic burden of medication error in England. BMJ Qual Saf. 2021;30(2):96-105. doi:10.1136/bmjqs-2019-010206

10. Lainer M, Mann E, Sönnichsen A. Information technology interventions to improve medication safety in primary care: a systematic review. Int J Qual Heal Care. 2013;25(5):590-598. doi:10.1093/ intqhe/mzt043

11. Ney JP, Weathers AL. Computerized prescriber order entry and opiate prescription in ambulatory care visits. $J$ Am Pharm Assoc. 2019;59 (2):S52-S56. doi:10.1016/j.japh.2019.01.010

12. Miller RJH, Bell A, Aggarwal S, Eisner J, Howlett JG. Computerized Electronic Order Set: use and Outcomes for Heart Failure Following Hospitalization. CJC Open. 2020;2(6):497-505. doi:10.1016/j. cjco.2020.06.009

13. Webair HH, Al-Assani SS, Al-Haddad RH, Al-Shaeeb WH, Bin Selm MA, Alyamani AS. Assessment of patient safety culture in primary care setting, Al-Mukala, Yemen. BMC Fam Pract. 2015;16(1):1-10. doi:10.1186/s12875-015-0355-1

14. Peters M, Godfrey C, McInerney P, Munn Z, Tricco A, Khalil H. Chapter 11: scoping Reviews (2020 version). In: aromataris E, Munn Z (Editors). JBI Man Evid Synth. 2020. doi:10.46658/JBIMES-20-12

15. Kamioka H. Preferred reporting items for systematic review and meta-analysis protocols (prisma-p) 2015 statement. Japanese Pharmacol Ther. 2019;47(8):1177-1185.

16. Centre for Evidence-Based Medicine. EBM Tools: study Design. University of Oxford; 2021. Available from: https://www.cebm.ox. ac.uk/resources/ebm-tools/study-designs. Accessed July 8, 2021.

17. Madlabana CZ, Mashamba-Thompson TP, Petersen I. Performance management methods and practices among nurses in primary health care settings: a systematic scoping review protocol. Syst Rev. 2020;9 (1):1-9. doi:10.1186/s13643-020-01294-w

18. Fischer SH, Rudin RS, Shi Y, et al. Trends in the use of computerized physician order entry by health-system affiliated ambulatory clinics in the United States, 2014-2016. BMC Health Serv Res. 2020;20 (1):1-6. doi:10.1186/s12913-020-05679-4

19. Dhavle AA, Yang Y, Rupp MT, Singh H, Ward-Charlerie S, Ruiz J. Analysis of prescribers' notes in electronic prescriptions in ambulatory practice. JAMA Intern Med. 2016;176(4):463-470. doi:10.1001/ jamainternmed.2015.7786 
20. Chackunkal E, Dhanapal Vogel V, Grycki M, Kostoff D. Improving adherence to the Epic Beacon ambulatory workflow. J Oncol Pharm Pract. 2017;23(4):273-277. doi:10.1177/1078155216637215

21. Motulsky A, Liang MQ, Moreault MP, Borycki E, Kushniruk A, Sicotte C. Evaluation of a nationwide e-prescribing system. Stud Health Technol Inform. 2019;264:714-718. doi:10.3233/SHT I190316

22. Bulut S, Y1ldiz A, Kaya S. Evaluation of transition to electronic prescriptions in Turkey: perspective of family physicians. Int $J$ Heal Policy Manag. 2019;8(1):40-48. doi:10.15171/ijhpm.2018.89

23. Donabedian A. Evaluating The Quality of Medical Care. Milbank. 2005;83(4):691-729. doi:10.1111/j.1468-0009.2005.00397.x

24. Aldughayfiq B, Sampalli S. Digital Health in Physicians' and Pharmacists' Office: a Comparative Study of e-Prescription Systems' Architecture and Digital Security in Eight Countries. Omi a J Integr Biol. 2021;25(2):102-122. doi:10.1089/omi.2020.0085

25. Gimenes FRE, Miasso AI, De Lyra DP, Grou CR. Electronic prescription as contributing factor for hospitalized patients' safety. Pharm Pract. 2006;4(1):13-17. doi:10.4321/S1885-642X2006000100003

26. Abramson E, Patel V, Pfoh E, Kaushal R. How Physician Perspectives on E-Prescribing Evolve over Time. Appl Clin Inform. 2016;07(04):994-1006. doi:10.4338/aci-2016-04-ra-0069

27. Craxford S, Taylor L, Duguid A, Shivji F, Pickering S. The learning curve of electronic prescribing. Br J Hosp Med. 2015;76(9):538-540. doi:10.12968/hmed.2015.76.9.538
28. Ai A, Wong A, Amato M, Wright A. Communication failure: analysis of prescribers' use of an internal free-text field on electronic prescriptions. $J$ Am Med Informatics Assoc. 2018;25(6):709-714. doi:10.1093/jamia/ ocy003

29. Allen AS, Sequist TD. Pharmacy dispensing of electronically discontinued medications. Ann Intern Med. 2020;172(1):ITC1-ITC14. doi:10.7326/AWED202001070

30. Al-Kahtani NK, Ramzi OI, Subbarayalu AV, Almulhim JA, Almulhim BF. Physicians perception toward an electronic prescribing system at an academic medical center (Amc) In Saudi Arabia: an exploratory study. Int J Sci Technol Res. 2019;8(10):358-363.

31. Oktarlina RZ. E-prescribing: benefit, barrier, and adopting challenge in electronic prescribing. $J$ Med. 2020;21(2):98-101. doi:10.3329/ jom.v21i2.50213

32. Imlach F, Mckinlay E, Kennedy J, et al. E - prescribing and access to prescription medicines during lockdown: experience of patients in Aotearoa/New Zealand. BMC Fam Pract. 2021;22:1-12. doi:10.1186/s12875-021-01490-0

33. Eriksen II, Melberg HO. The effects of introducing an electronic prescription system with no copayments. Health Econ Rev. 2015;5 (1):1-8. doi:10.1186/s13561-015-0056-4

34. Esmaeil Zadeh P, Tremblay MC. A review of the literature and proposed classification on e-prescribing: functions, assimilation stages, benefits, concerns, and risks. Res Soc Adm Pharm. 2016;12 (1):1-19. doi:10.1016/j.sapharm.2015.03.001
Journal of Multidisciplinary Healthcare

\section{Publish your work in this journal}

The Journal of Multidisciplinary Healthcare is an international, peerreviewed open-access journal that aims to represent and publish research in healthcare areas delivered by practitioners of different disciplines. This includes studies and reviews conducted by multidisciplinary teams as well as research which evaluates the results or conduct of such teams or healthcare processes in general. The journal covers a very wide range of areas and welcomes submissions from practitioners at all levels, from all over the world. The manuscript management system is completely online and includes a very quick and fair peer-review system. Visit http://www.dovepress.com/testimonials. php to read real quotes from published authors. 